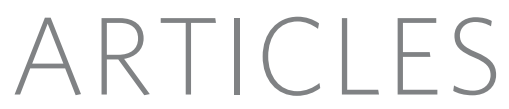

\title{
Tetherin inhibits retrovirus release and is antagonized by HIV-1 Vpu
}

\author{
Stuart J. D. Neil ${ }^{1}$, Trinity Zang $^{1} \&$ Paul D. Bieniasz ${ }^{1}$
}

Human cells possess an antiviral activity that inhibits the release of retrovirus particles, and other enveloped virus particles, and is antagonized by the HIV-1 accessory protein, $\mathrm{Vpu}$. This antiviral activity can be constitutively expressed or induced by interferon- $\alpha$, and it consists of protein-based tethers, which we term 'tetherins', that cause retention of fully formed virions on infected cell surfaces. Using deductive constraints and gene expression analyses, we identify CD317 (also called BST2 or HM1.24), a membrane protein of previously unknown function, as a tetherin. Specifically, CD317 expression correlated with, and induced, a requirement for Vpu during HIV-1 and murine leukaemia virus particle release. Furthermore, in cells where HIV-1 virion release requires Vpu expression, depletion of CD317 abolished this requirement. CD317 caused retention of virions on cell surfaces and, after endocytosis, in CD317-positive compartments. Vpu co-localized with CD317 and inhibited these effects. Inhibition of Vpu function and consequent mobilization of tetherin's antiviral activity is a potential therapeutic strategy in HIV/AIDS.

Some human cells possess a poorly defined antiviral activity, the existence of which is indicated by studies of the HIV-1 accessory protein, $\mathrm{Vpu}$, that inhibits the release of certain enveloped virus particles. Specifically, Vpu is required for efficient HIV-1 particle release in certain human cells ${ }^{1-3}$, and HeLa cells are a prototypic example of cells that exhibit this requirement ${ }^{4}$. However, $\mathrm{Vpu}$ is completely dispensable for efficient HIV-1 particle release in some other human cells and in the African green monkey cell line COS-7 $7^{5,6}$. Notably, fusion of HeLa cells with COS-7 cells results in heterokaryons that exhibit the properties of $\mathrm{HeLa}$ cells ${ }^{7}$, suggesting the existence of an inhibitor of virion release that is expressed in HeLa cells, but not in COS cells, and is antagonized by Vpu. Moreover, Vpu stimulates the release of retrovirus particles as diverse as HIV-1 and murine leukaemia virus (MLV) from HeLa cells ${ }^{4}$, as well as virus-like particles based on the structural protein of Ebola virus ${ }^{8}$, further suggesting that the inhibitor works in a nonspecific way to block envelope virus particle release.

Early analyses of cells infected with Vpu-deleted HIV-1 strains revealed that virions accumulate within intracellular vacuoles and at the cell surface, at the expense of particle release ${ }^{3,4}$. Recent work has shown that the intracellular virions are primarily localized in $\mathrm{CD} 3^{+}$endosomes ${ }^{6,8}$, and that they accumulate at this location as a result of their internalization from the cell surface ${ }^{6,8-10}$. Moreover, HIV-1 particles that are retained by cells owing to the absence of Vpu are fully formed and mature, and can be released from cell surfaces by protease, particularly when their endocytosis is blocked ${ }^{6,8}$. Thus, the inhibitor that is counteracted by Vpu seems to induce adherence of nascent virions to the surface of cells, after completion of their assembly, and from where they may be internalized. We recently found that a requirement for Vpu during HIV-1 virion and Ebolavirus-like particle release can be induced or enhanced in fibroblasts or $\mathrm{T}$ cells by treatment with interferon- $\alpha(\text { IFN- } \alpha)^{8}$. Notably, the nature of the HIV-1 release defect that accompanies Vpu deletion in these IFN- $\alpha$-treated cells precisely recapitulates that observed constitutively in HeLa cells ${ }^{6,8}$. Thus, these data suggest that the inhibitor that is antagonized by $\mathrm{Vpu}$ is an IFN- $\alpha$-induced, cell-surfaceprotein-based tether, that we term 'tetherin'. However, although evidence for the existence of tetherin is quite considerable, it is indirect, and the identity of tetherin is unknown.

\section{Identification of tetherin candidates}

To identify tetherin candidates we applied several constraints based on the aforementioned studies that predicted its distribution and nature. First, tetherin should be constitutively expressed in a cell line (HeLa) where HIV-1 Vpu is constitutively required for efficient retrovirus particle release ${ }^{4}$, but not in cells where $\mathrm{Vpu}$ is dispensable (for example, HOS, 293T or HT1080 cells ${ }^{6,8}$; Supplementary Table 1). Second, because a requirement for Vpu during HIV-1 release becomes evident after IFN- $\alpha$ treatment in 293T and HT1080 cells (Supplementary Table 1 ) and is enhanced by IFN- $\alpha$ treatment in Jurkat cells and primary lymphocytes ${ }^{8}$, then tetherin expression should reflect this property. Moreover, because Vpu-defective HIV-1 release seems to be blocked after the fission of cell and virion membranes ${ }^{3,4,6}$, tetherin should be a secreted or membrane-associated protein. Although none of the aforementioned criteria alone would provide sufficient discriminatory power to identify tetherin, we reasoned that their simultaneous application might reduce candidates to a reasonable number to be tested individually.

Microarray analyses of messenger RNAs expressed in the aforementioned untreated and IFN- $\alpha$-treated cell lines revealed few $(<10)$ candidates that conformed to the above criteria. Among these was CD317, a membrane protein of unknown function ${ }^{11}$, as well as three 'interferon-induced transmembrane proteins', IFITM1, IFITM2 and IFITM3 (Fig. 1a and Supplementary Table 2). On the basis of their expression levels in the various cell lines, CD317 appeared to be the most compelling of these candidates (Fig. 1a and Supplementary Table 2). Indeed, its expression, as measured by microarray or quantitative reverse transcription-polymerase chain reaction (qRT-PCR) analysis, was $>20$-fold higher in HeLa than in HOS cells, was induced $>20$-fold by IFN- $\alpha$ in 293 T and HT1080 cells, and was induced two- to fivefold in Jurkat cells (Fig. 1b, c). Moreover, IFN- $\alpha$ treatment accentuates the requirement for Vpu in HIV-1 replication in primary lymphocytes ${ }^{8}$ and, concordantly, CD317 mRNA was 
induced by IFN- $\alpha$ in $\mathrm{CD}^{+}$enriched lymphocytes from two donors (Fig. 1d).

Transfection of 293T cells with wild-type (HIV-1(WT)) and Vpudeleted (HIV-1(delVpu)) proviral plasmids generated, as expected, equivalent yields of HIV-1 particles, determined using western blot or infectious virion measurements (Fig. 1e, f). However, HIV-1 (delVpu), but not HIV-1(WT), virion production was strongly inhibited by co-expressed CD317. Notably, Gag protein expression or processing was not affected by CD317 (Fig. 1e), suggesting that the candidate tetherin indeed inhibited particle release. Conversely, similar transient transfection assays revealed that overexpression of IFITM1, IFITM2 or IFITM3 did not affect HIV-1(delVpu) release (data not shown).

\section{Inhibition of retrovirus release by tetherin}

Because Vpu affects CD4 expression as well as virion release ${ }^{12}$ and because CD4 can interfere with virus release and infectivity ${ }^{13,14}$, the effect of the candidate tetherin on HIV-1(WT) and HIV-1(delVpu) replication was analysed using single-cycle HIV-1 replication assays in $\mathrm{CD}^{-}$cells. These assays were done in cells that do (HeLa) or do not (293T, HT1080) constitutively express CD317. Furthermore, derivatives of 293T and HT1080 cells that stably expressed CD317 were established via retroviral vector transduction, and qRT-PCR analyses revealed that CD317 mRNA was only moderately overexpressed ( $\sim 2-6$-fold more abundant than in HeLa cells) in these cell lines. Crucially, stable CD317 expression reduced Vpu-defective HIV-1 virion yield by $>100$-fold (293T cells) and $\sim 80$-fold (HT1080 cells) over a single replication cycle (Fig. 2a-d). Conversely, CD317 had only marginal effects ( $\sim 2$-fold) on HIV-1(WT) yield. Viral Gag protein expression in HIV-1(WT)- or HIV-1(delVpu)-infected 293T or HT1080 cells was equal in the presence or absence of CD317 (Fig. 2c, d), indicating that all preceding steps in HIV-1 replication were unaffected and that CD317 specifically inhibited HIV-1 virion release. In addition, transfection of $293 \mathrm{~T}$ cells with vectors expressing MLV GagPol revealed that stable expression of CD317 markedly reduced the yield of MLV particles, without affecting MLV Gag expression (Fig. 2e). Moreover, inhibition of MLV particle release by CD317 was almost completely abolished when Vpu was coexpressed with MLV GagPol. (Fig. 2e).

In single-cycle HIV-1 replication assays, conducted in HeLa cells that constitutively express CD317, HIV-1(delVpu) virions were released approximately 20-fold less efficiently than HIV-1(WT) counterparts (Fig. 2f, g). To determine whether CD317 was required for this phenotype, the same assay was done using HeLa cells transfected with a CD317-targeted short interfering RNA (siRNA) pool that effectively knocked down expression of the corresponding haemagglutinin (HA)-tagged protein (Supplementary Fig. 1), or a control siRNA pool. CD317-targeted siRNAs, but not control siRNAs, enhanced the yield of HIV-1(delVpu) from HeLa cells by $\sim 13$-fold, but had negligible effects on HIV-1(WT) yield (Fig. 2g). Notably,
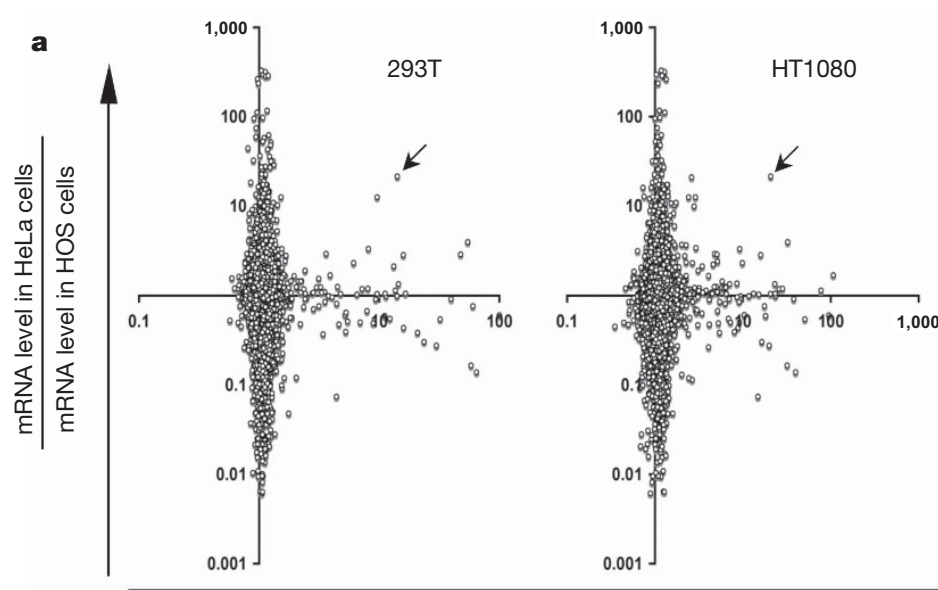

Fold induction by IFN- $\alpha$
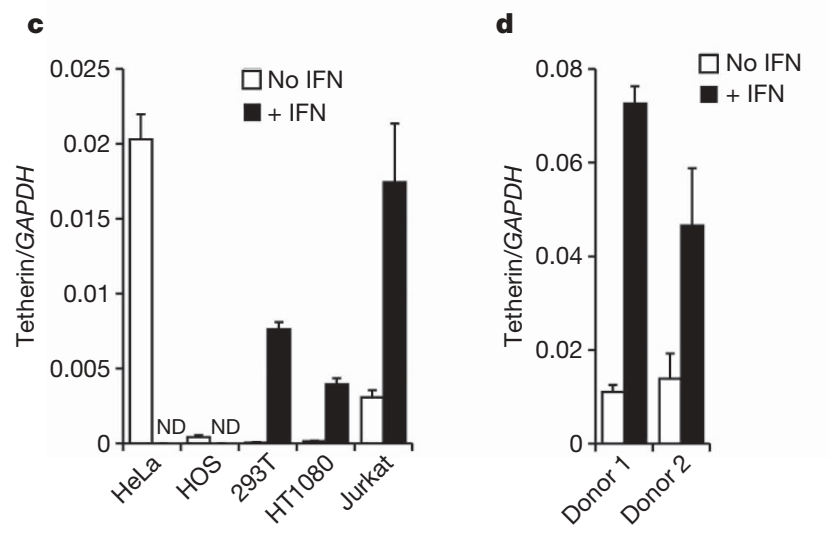

Figure 1 | Identification of CD317 as a candidate tetherin. a, Gene expression analysis of cells in which Vpu is, or is not, required for HIV-1 virion release. The ratio of mRNA levels in HeLa cells divided by those in HOS cells ( $y$ axis) is plotted against levels in IFN- $\alpha$-treated 293T, HT1080 or Jurkat cells divided by those in corresponding untreated cells ( $x$ axis). Candidate tetherins appear in the upper right quadrant; arrows indicate $C D 317 . \mathbf{b}$, levels of $C D 317$ mRNA (arbitrary units) as measured in the array analysis. ND, not done. c, d, Levels of the candidate tetherin mRNA in cell
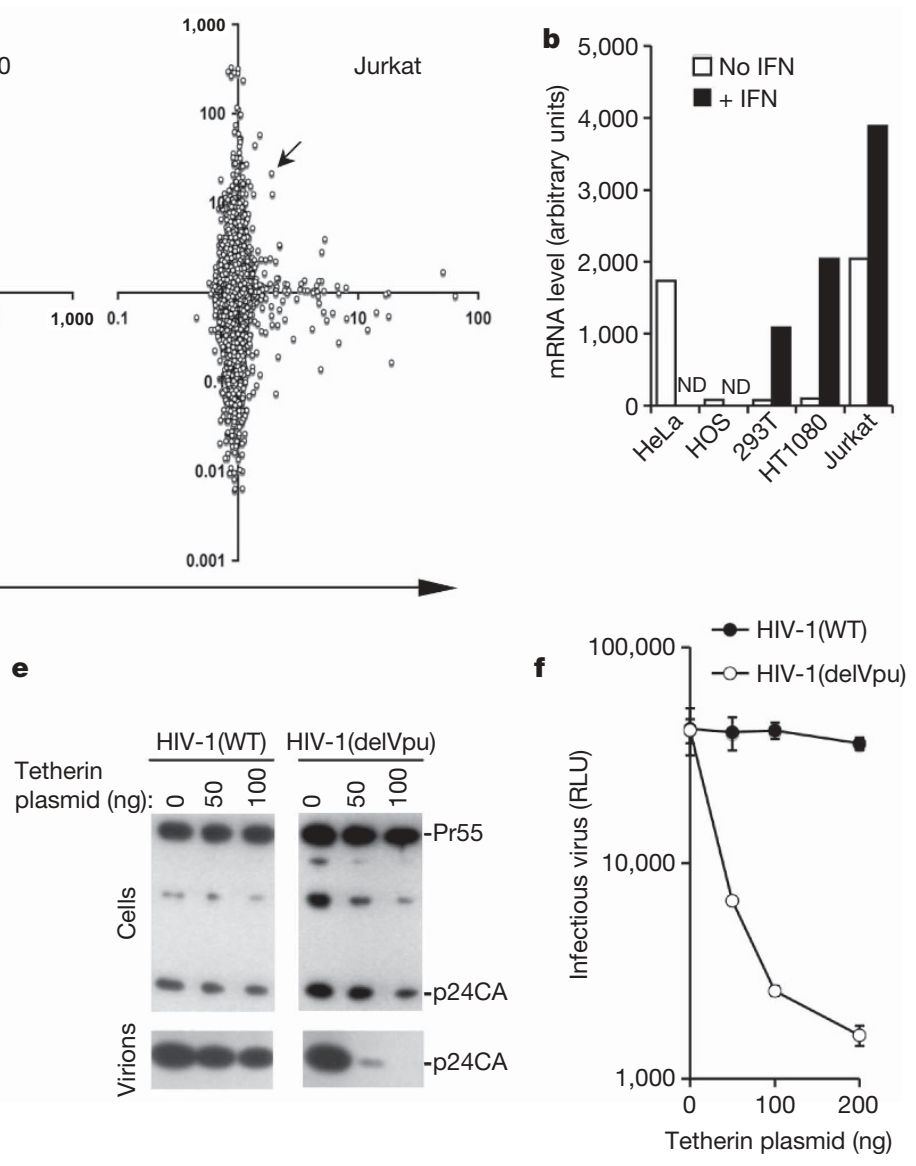

lines (c) or $\mathrm{CD}^{+}{ }^{+} \mathrm{T}$ cells $(\mathrm{d})$, presented as mRNA copies per copy of GAPDH ( \pm s.d., $n=3$ ), as measured by qRT-PCR. e, Western blot analysis (anti-p24 capsid) of 293T cell lysates and virions after co-transfection with HIV-1(WT) and HIV-1(delVpu) proviral plasmids and varying amounts of CD317 (untagged) expression plasmid. f, Same as e, except that infectious virus yield was measured using TZMbl indicator cells and expressed as relative light units (RLU) ( \pm s.d., $n=3$ ). 
CD317 knockdown had no effect on Gag expression and processing (Fig. 2f); rather it relieved a requirement for Vpu during particle release.

CD317 (referred to hereafter as tetherin) has an unusual topology, in that it harbours an amino-terminal cytoplasmic tail, a singlemembrane-spanning sequence, a predicted extracellular coiled-coil domain and a putative carboxy-terminal glycosyl phosphatidylinositol (GPI) membrane anchor ${ }^{15,16}$ (Fig. 3a). All of the aforementioned assays were done using an authentic, untagged version of tetherin.
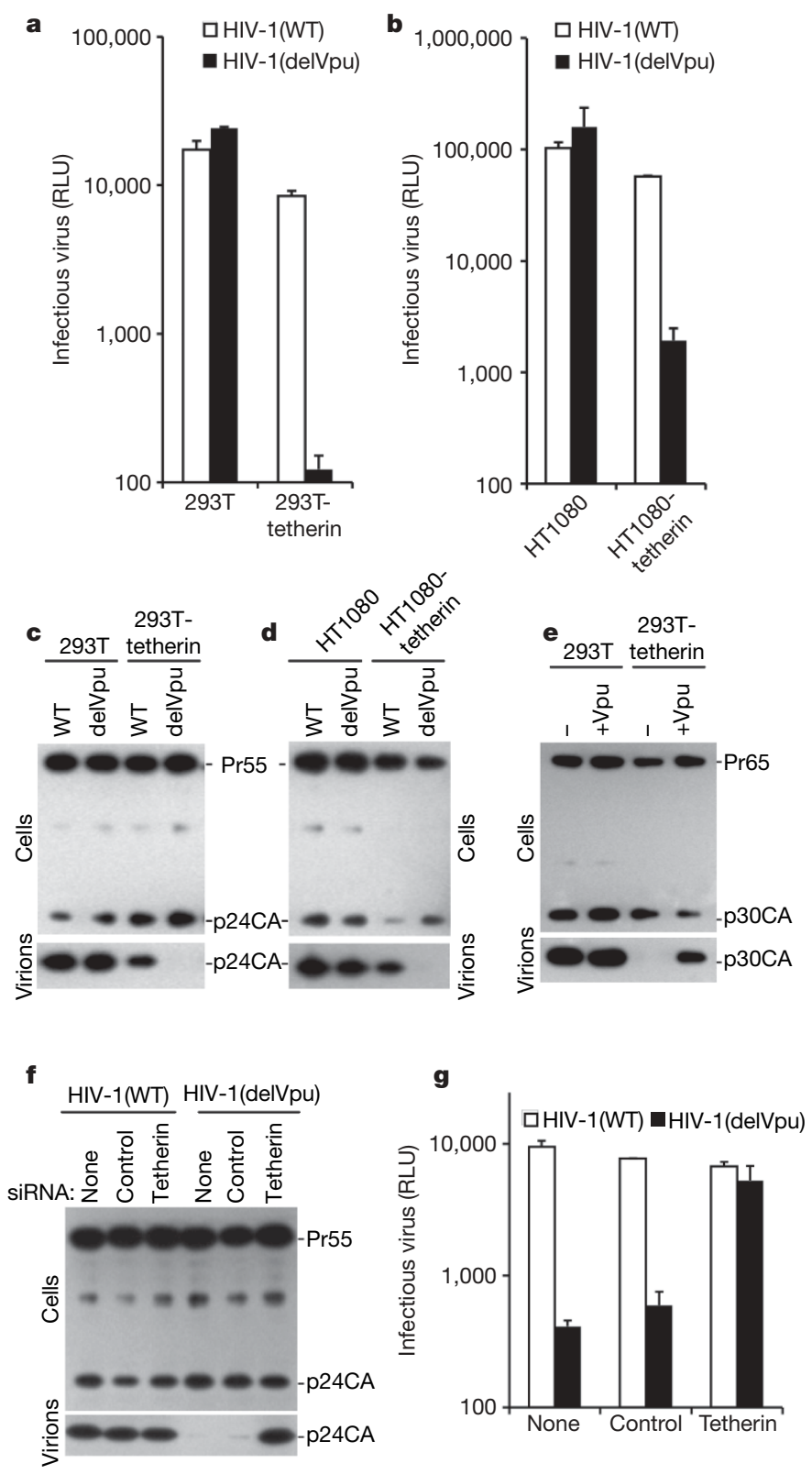

Figure 2 | Tetherin blocks HIV-1 virion release and is necessary to impose a requirement for $\mathbf{V p u}$. $\mathbf{a}, \mathbf{b}$, Infectious virion yield ( \pm s.d., $n=3$ ), measured as in Fig. 1, from 293T cells (a) or HT1080 cells (b) that were unmodified or stably expressed untagged tetherin, after a single cycle of HIV-1(WT) or HIV-1 (delVpu) replication. c, d, Same as for $\mathbf{a}$ and b, except that western blot analysis of cell lysates along with progeny virions (anti-p24 capsid) was done. e, Western blot analysis of cell lysates along with progeny virions (anti-p30 capsid) after expression of MLV GagPol, in the absence or presence of Vpu, in $293 \mathrm{~T}$ cells that were unmodified or stably expressed untagged tetherin. f, Western blot analysis (anti-p24 capsid) of HeLa cells and progeny virions during a single cycle of HIV-1 replication after transfection with no siRNAs (none), control or tetherin-specific siRNAs. g, Same as f, except that infectious virion yield ( \pm s.d., $n=3$ ) was measured, as in Fig. 1 .
However, to facilitate more detailed analyses, an N-terminally HAepitope-tagged tetherin derivative was generated, and this protein accurately recapitulated the antiviral properties of the untagged molecule (Fig. 3b, c). HA-tetherin exhibited a complex pattern of distribution in cells, suggesting that it localized to multiple membranous compartments, including the plasma membrane (Supplementary Fig. 2). Moreover, tetherin migrated as several species in SDS-PAGE analyses, presumably as a result of heterogeneous glycosylation $^{11}$ (Fig. 3b). A tetherin variant with a truncated $\mathrm{N}$-terminal cytoplasmic tail (delCT) migrated as a single species in SDS-PAGE analyses, and its subcellular distribution was aberrant, suggesting that it remained non-glycosylated or uniformly glycosylated, and failed to complete proper transport through the secretory pathway (Fig. 3b and Supplementary Fig. 2). Notably, tetherin(delCT) did not affect HIV-1(delVpu) particle release (Fig. 3b, c). Removal of the putative GPI modification signal in tetherin, (delGPI), increased tetherin protein levels but did not overtly alter glycosylation or subcellular distribution (Fig. 3b and Supplementary Fig. 2). However, removal of the putative GPI anchor completely abolished tetherin's ability to inhibit HIV-1(delVpu) release (Fig. 3b, c).

\section{Effects of tetherin on nascent HIV-1 particles}

In circumstances where HIV-1 release requires Vpu expression, Vpudefective HIV-1 virions assemble and mature, but remain tethered to the surface of cells ${ }^{3,4,6}$. Thereafter, a proportion of virions are internalized and accumulate in endosomes ${ }^{6,9,10}$. To determine whether tetherin induced this phenotype, 293T cells were transiently co-transfected with plasmids expressing HIV-1 Gag-GFP, tetherin and/or Vpu. Tetherin markedly increased the proportion of cells that contained prominent intracellular accumulations of Gag-GFP puncta, which were often associated with $\mathrm{CD}^{+}{ }^{+}$endosomes (Fig. 4a, b and Supplementary Fig. 3a). Conversely, a mutant tetherin (delGPI) that did not inhibit HIV-1(delVpu) release did not affect the distribution of Gag-GFP (Supplementary Fig. 3a). Importantly, tetherin did not induce intracellular accumulation of Gag-GFP when Vpu was co-expressed (Fig. 4a and Supplementary Fig. 3a). Moreover, accumulation of Gag-GFP puncta in the endosomes of 293T cells stably expressing tetherin was blocked by a dominant-negative
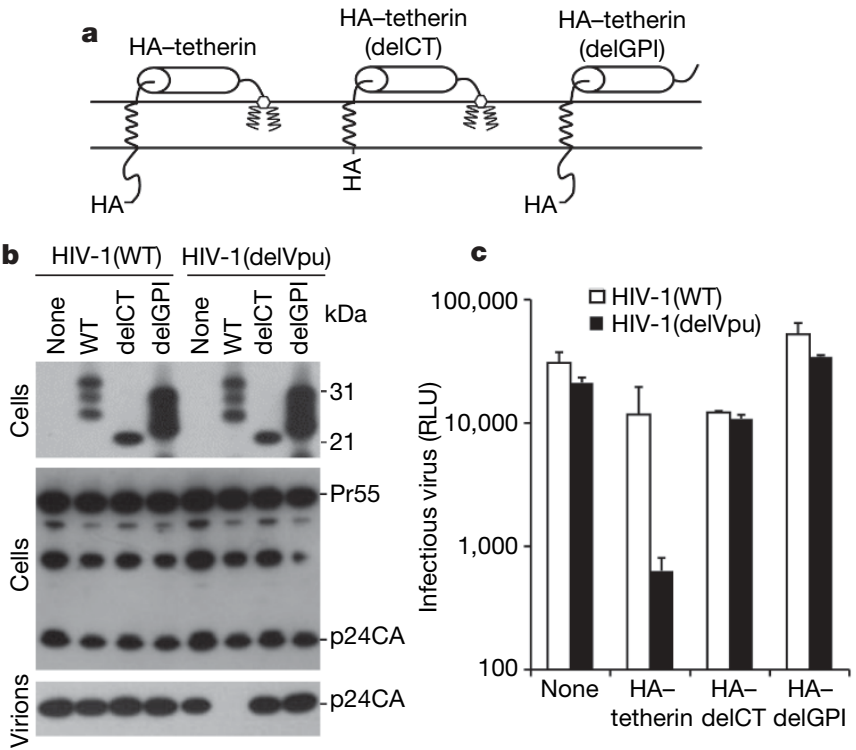

Figure 3 | Effects of HA-tagged tetherin and mutants on HIV-1 release. a, Schematic representation of the HA-tagged tetherin and mutants. b, Western blot analysis (anti-HA, upper panel; p24 capsid, centre and lower panels) of cells and virions after transfection of 293T cells with proviral plasmids and plasmids expressing HA-tagged tetherin molecules. c, Virion yield from the same cells as in $\mathbf{b},( \pm$ s.d., $n=3$ ) measured using infectivity assays. 
Rab5a mutant (S34N) as well as by Vpu (Fig. 4c and Supplementary Fig. 3b, c), suggesting that tetherin-induced association of Gag-GFP with $\mathrm{CD} 3^{+}$compartments occurred via endocytic uptake of nascent particles from the plasma membrane. Consistent with this notion, when swollen endosomes were induced by expression of a dominantactive Rab5a mutant (Q79L), tetherin induced the appearance of Gag-GFP puncta within the swollen endosomal lumen (Fig. 4c). Similar experiments, done in HT1080 cells stably expressing tetherin and infected with an HIV-1 strain carrying YFP inserted into the stalk region of the Gag matrix domain (HIV/MA-YFP) ${ }^{17,18}$, showed that YFP-positive puncta accumulated in intracellular compartments of
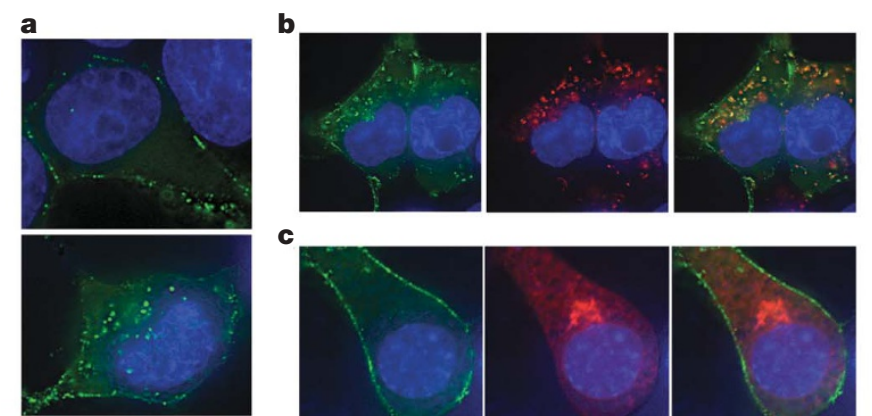

c
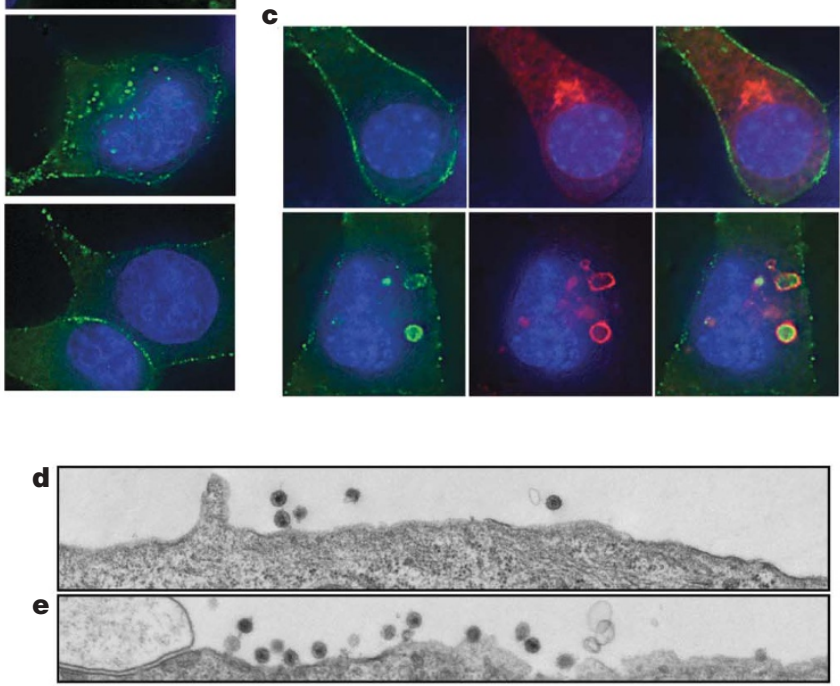

$\mathbf{f}$

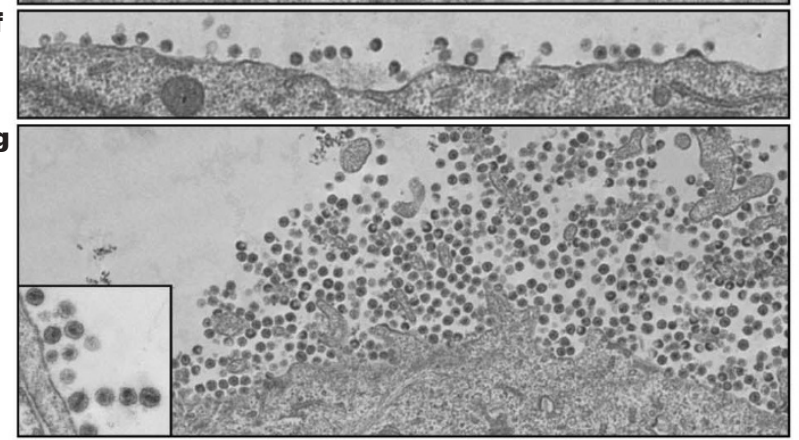

h

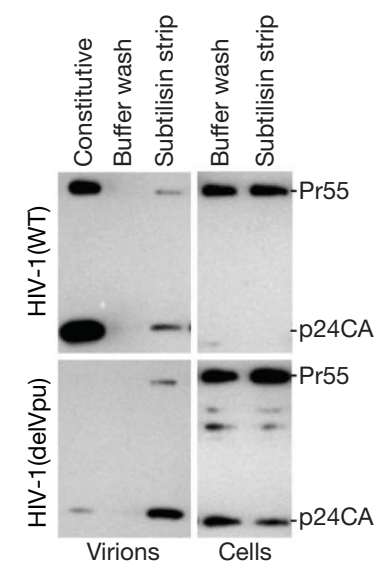

+ Cherry-FP-Rab5a(S34N)

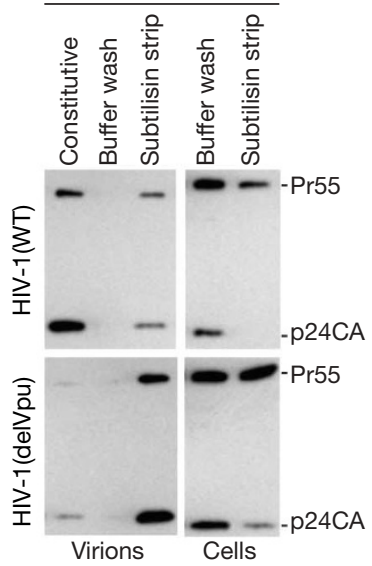

tetherin-expressing cells, specifically when a Vpu-deleted HIV/MAYFP virus was used (Supplementary Fig. 3d).

Electron microscopic analysis of unmodified HT1080 cells, infected with HIV-1(WT) or HIV-1(delVpu), revealed occasional budding structures and mature virions associated with the plasma membrane (Fig. 4d, e). In HT1080 cells stably expressing tetherin and infected with HIV-1(WT), virions were found associated with the plasma membrane at marginally greater frequency (Fig. 4f). In stark contrast, HIV-1(delVpu) infection of tetherin-expressing cells often resulted in massive accumulations of mature virion particles on cell surfaces (Fig. 4g and Supplementary Fig. 4). Mature HIV-1(delVpu) virions were also frequently observed in intracellular compartments in tetherin-expressing HT1080 cells (Supplementary Fig. 4), consistent with the notion that the particles retained by tetherin can be endocytosed (Fig. 4b, c).

To determine whether tetherin caused protein-mediated tethering of HIV-1 to cell surfaces, we used an assay in which tethered virions are recovered from cell surfaces by protease 'stripping'6,8. In HIV-1transfected or -infected $293 \mathrm{~T}$ cell cultures, the majority of virions that can be recovered are constitutively released into the extracellular medium, irrespective of $\mathrm{Vpu}$ expression, and only a minor fraction of the total virion output is recovered by protease stripping of cell surfaces $^{8}$. Similarly, In 293T cells stably expressing tetherin, HIV-1 (WT) virions were also predominantly constitutively released and only a minor fraction were recovered by protease stripping (Fig. 4h). Conversely, recovery of the majority of HIV-1(delVpu) virions required protease stripping from the surface of tetherinexpressing 293T cells. In similar experiments, co-expression of Rab5a(S34N) slightly reduced the overall production of HIV-1 (WT) and HIV-1(delVpu). However, presumably because virion endocytosis was blocked (Fig. 4c), the recovery of HIV-1(delVpu) by protease stripping of the tetherin-expressing cells' surface was enhanced and, in fact, equalled the yield of constitutively released HIV-1(WT) virions (Fig. 4h).

\section{Localization of HIV-1 Gag, Vpu and tetherin}

Notably, the intracellular compartments in which Gag-GFP or HIV/ MA-YFP puncta accumulated in transfection or infection assays were often HA-tetherin-positive (Fig. 5a and Supplementary Fig. 5). Moreover, YFP puncta that were localized at the surface of HIV/MA-YFP(delVpu)-infected cells were also often positive for HA-tetherin (Supplementary Fig. 5). It is possible, therefore, that tetherin is directly responsible for the retention of nascent virions on cell surfaces and their movement into intracellular compartments. Notably, association of Gag-GFP or HIV/MA-YFP puncta with HAtetherin was prevented by co-expression of $\mathrm{Vpu}$ (Fig. 5a and Supplementary Fig. 5), and Vpu itself co-localized almost perfectly with HA-tetherin (Fig. 5b). Although Vpu did not have overt effects on tetherin levels or subcellular distribution (Fig. 3b, Fig. 5b and data not shown), only a minor fraction of HA-tetherin was associated

Figure 4 | Tetherin expression in the absence of Vpu causes virion retention on cell surfaces and virion endocytosis. a, Examples of Gag-GFP localization in 293T cells when expressed alone (top panel), or co-expressed with HA-tetherin (centre panel) or HA-tetherin and Vpu (bottom panel). b, Gag-GFP (green) localization in 293T cells stably expressing untagged tetherin and stained with anti-CD63 (red). c, Localization of Gag-GFP (green) and either cherry-FP-Rab5(S43N) (top row, red) or cherryFP-Rab5(Q79L) (bottom row, red) in 293T cells stably expressing untagged tetherin. In b and c, the right panel is a merged image. $\mathbf{d - g}$, Electron micrographs showing plasma membranes of unmodified HT1080 cells (d, e) or HT1080 cells expressing untagged tetherin $(\mathbf{f}, \mathbf{g})$ after infection with HIV-1(WT) (d, f) or HIV-1(delVpu) (e, g). The inset shows an expanded view of plasma-membrane-associated virions. $\mathbf{h}$, Western blot analysis of virions that were constitutively released from 293T cells stably expressing untagged tetherin, or released after buffer wash or subtilisin stripping, after transfection with HIV-1(WT) or HIV-1(delVpu) proviral plasmids, alone or with cherry-FP-Rab5(S43N) co-expression. 
with the plasma membrane, thus its potential removal from that site was difficult to evaluate. Notably, an inactive Vpu mutant in which the transmembrane domain was replaced with that of CD8 $(\mathrm{Vpu}(\mathrm{CD} 8 \mathrm{TM}))^{6}$ did not co-localize extensively with HA-tetherin (Fig. 5b). Conversely, a Vpu mutant whose activity was partly impaired by truncation of its cytoplasmic tail at residue 50 (Vpu(1-50), Supplementary Fig. 6) co-localized extensively, albeit incompletely, with HA-tetherin, and caused the distribution of tetherin to be more diffuse and plasma-membrane associated (Fig. 5b). Thus, these results suggest a direct or indirect association between tetherin and $\mathrm{Vpu}$, perhaps involving the Vpu transmembrane domain.

\section{Discussion}

Overall, tetherin specifically inhibited retrovirus particle release in the absence of $\mathrm{Vpu}$ and its expression precisely recapitulated the
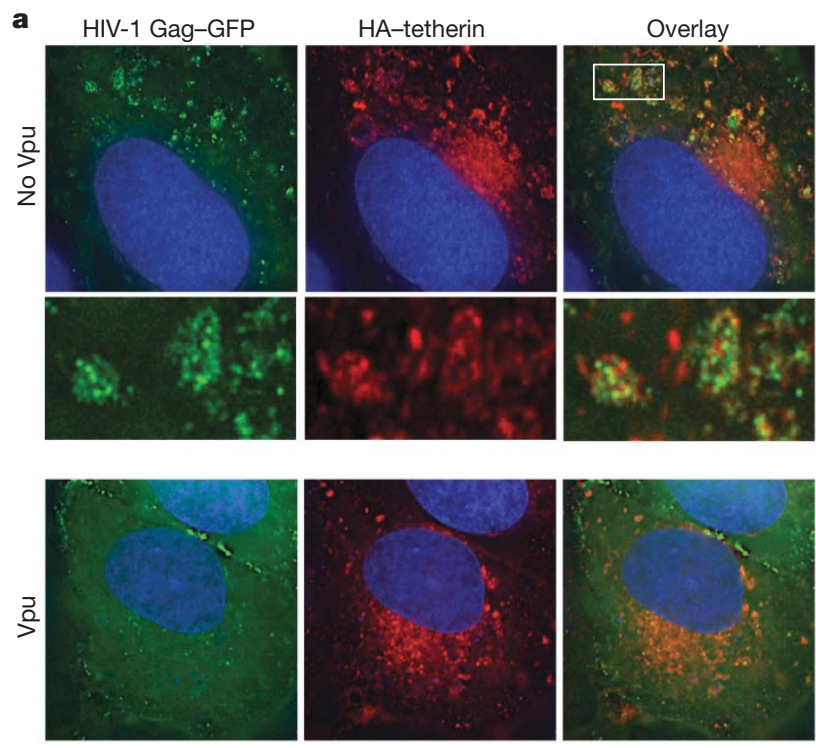

b
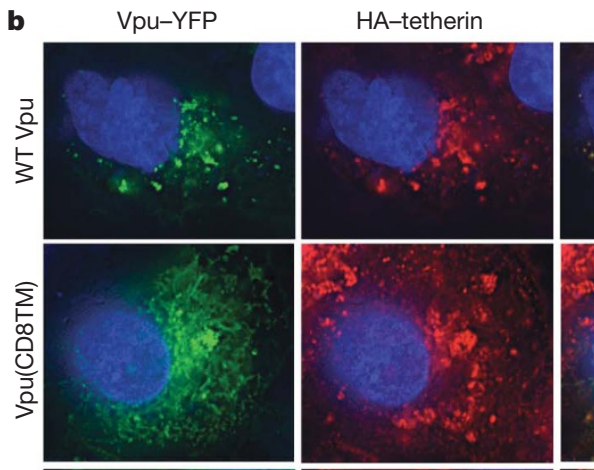

Overlay
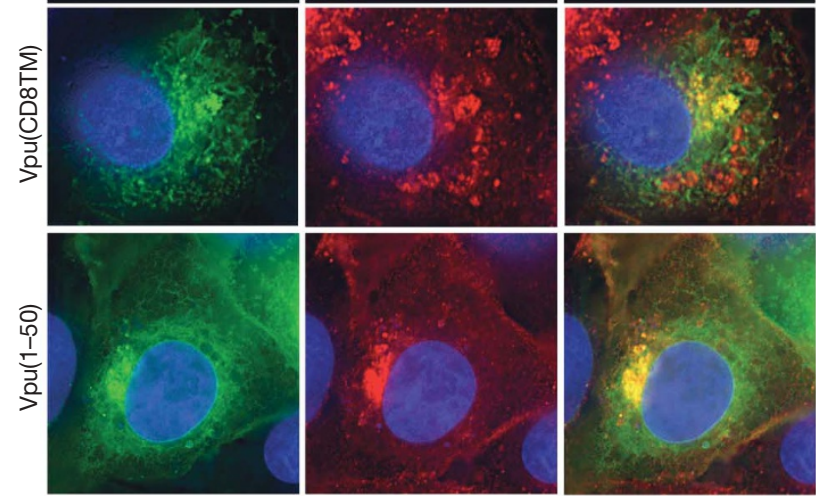

Figure 5 | Tetherin localization in relation to HIV-1 Gag and Vpu.

a, Localization of transiently expressed Gag-GFP and stably expressed HA-tetherin in HT1080 cells in the absence (top panels) or presence (bottom panels) of co-expressed Vpu. An expanded view of a portion of the top panel (white box) is also shown (centre panels). b, Localization of transiently expressed wild-type Vpu-YFP (top row), Vpu(CD8TM) (centre row) or $\mathrm{Vpu}(1-50)$ (bottom row) and stably expressed HA-tetherin (red) in HT1080 cells. characteristic HIV-1 particle release defects that have previously been associated with loss of $\mathrm{Vpu}$ function ${ }^{2-4,6,8,9}$. Moreover, tetherin co-localized with Vpu and, when Vpu was absent, virions were associated with tetherin at the cell surface and in intracellular compartments. These findings strongly suggest that a major function of the HIV-1 Vpu protein is to facilitate virus spread by antagonizing tetherin-induced particle retention. Notably, previous work has shown that $\mathrm{Vpu}$ can enhance the release of a wide range of retroviruses, as well as Ebola virus, in HeLa cells or other cells treated with IFN- $\alpha^{4,8}$, suggesting that tetherin acts in a rather nonspecific way to inhibit enveloped virion release. This prediction is borne out by our findings that tetherin can indeed inhibit the release of MLV as well as HIV-1. At present, it is unclear whether only the human form of tetherin has this antiviral function. Orthologues are present in other mammals, but $\mathrm{Vpu}$ is not required for efficient HIV-1 particle release from untreated African green monkey COS cells ${ }^{5}$, and fails to stimulate particle release that has been is inhibited by IFN- $\alpha$ treatment ${ }^{8}$. It will be interesting to determine whether these apparently hostspecies-specific differences in $\mathrm{Vpu}$ activity reflect divergence in tetherin sequence and function.

Tetherin is known to be expressed on terminally differentiated B cells $^{19}$, bone marrow stromal cells (hence the name BST2) ${ }^{11}$ and plasmacytoid dendritic cells ${ }^{20}$. Tetherin has also previously been shown to be broadly expressed on IFN- $\alpha$ induction ${ }^{19,20}$. Because tetherin is GPI-modified, it may partition into cholesterol-rich domains on the plasma membrane ${ }^{15}$. Potentially, this property could drive or facilitate encounters with assembling HIV-1 (or other enveloped virus) particles, which have a similar lipid composition ${ }^{21}$. Although we have not yet determined whether tetherin is actually incorporated into virion particles, the fact that electron microscopic analysis shows that, in the absence of $\mathrm{Vpu}, \mathrm{HIV}-1$ particles appeared to be tethered to each other as well as to cells suggests that this may be the case. Moreover, given tetherin's unusual topology, as well as its propensity to dimerize ${ }^{19}$, several possible configurations could be imagined in which tetherin might nonspecifically tether virions to cell membranes, and to each other (Supplementary Fig. 7).

Interestingly, tetherin protein levels are decreased by expression of the Kaposi's sarcoma herpesvirus K5 protein ${ }^{22}$. This fact, and the observations that tetherin inhibits divergent retroviruses and is widely expressed in the presence of IFN- $\alpha^{20}$, raises the possibility that tetherin might be an important component of the broad innate IFN$\alpha$-induced antiviral defence against enveloped viruses. Future work will determine the spectrum of viruses against which tetherin is active, precisely how tetherin induces virion retention, and how its action is counteracted by the HIV-1 Vpu protein.

\section{METHODS SUMMARY}

Microarray analyses. Total RNA from the various cell lines that were either untreated or treated with IFN- $\alpha$ was used to interrogate microarrays (Illumina). qRT-PCR. Total RNA from untreated or IFN- $\alpha$-treated cells was used to generate cDNA which was analysed using primer pairs for CD317 and GAPDH (Superarray Bioscience Corporation).

Tetherin expression constructs. Tetherin was transiently expressed using pCR3.1/HA-based plasmids or stably expressed using LHCX-based retroviral vectors. The delCT and delGPI variants of tetherin lacked the N-terminal 20 amino acids and C-terminal 19 amino acids, respectively.

Single-cycle HIV-1 replication and transfection-based virion release assays. 293T cells were transfected with wild-type (HIV-1(WT)) or Vpu-deleted (HIV-1 (delVpu)) proviral plasmids, along with tetherin expression plasmids. Alternatively, HeLa cells (that were either untransfected or transfected with siRNAs), 293T cells, or HT1080 cells were infected with VSV-G-pseudotyped HIV-1(WT) or HIV-1(delVpu). Thereafter, culture supernatants and infected cells were collected. Virus particle pellets collected from culture supernatants and corresponding cell lysates were analysed by western blot assays using anti-p24 monoclonal antibodies. Infectious virus yields were determined using HeLaTZMbl indicator cells.

Protease stripping assays. 293T cells stably expressing tetherin were transfected with proviral plasmids. Constitutively released particles or particles that were 
released after buffer wash or subtilisin stripping, as well as corresponding cell lysates, were analysed by western blotting.

Microscopy. Cells, plated on coverslips, were transfected with combinations of plasmids expressing HIV-1 Gag-GFP, tetherin, Vpu, and/or Rab5a mutants. Alternatively, cells were infected with VSV-G-pseudotyped HIV-1/MA-YFP. Anti-HA or anti-CD63 antibodies were used for staining. Microscopy was done using a Deltavision suite. For electron microscopy, HT1080 cells were infected with VSV-G-pseudotyped HIV-1(WT) or HIV-1(delVpu). Sections were stained with uranyl acetate and lead citrate and observed with a FEI G2 Tecnai TEM.

Full Methods and any associated references are available in the online version of the paper at www.nature.com/nature.

Received 14 November; accepted 17 December 2007.

Published online 16 January 2008; corrected 24 January 2008 (details online).

1. Terwilliger, E. F. et al. Functional role of human immunodeficiency virus type $1 \mathrm{vpu}$. Proc. Natl Acad. Sci. USA 86, 5163-5167 (1989).

2. Strebel, K. et al. Molecular and biochemical analyses of human immunodeficiency virus type 1 vpu protein. J. Virol. 63, 3784-3791 (1989).

3. Klimkait, T. et al. The human immunodeficiency virus type 1-specific protein vpu is required for efficient virus maturation and release. J. Virol. 64, 621-629 (1990).

4. Gottlinger, H. G. et al. Vpu protein of human immunodeficiency virus type 1 enhances the release of capsids produced by gag gene constructs of widely divergent retroviruses. Proc. Natl Acad. Sci. USA 90, 7381-7385 (1993).

5. Geraghty, R. J. et al. Cell type-dependence for Vpu function. J. Med. Primatol. 23, 146-150 (1994).

6. Neil, S. J. et al. HIV-1 Vpu promotes release and prevents endocytosis of nascent retrovirus particles from the plasma membrane. PLoS Pathog. 2, e39 (2006).

7. Varthakavi, $V$. et al. Viral protein $U$ counteracts a human host cell restriction that inhibits HIV-1 particle production. Proc. Natl Acad. Sci. USA 100, 15154-15159 (2003).

8. Neil, S. J. et al. An interferon- $\alpha$-induced tethering mechanism inhibits HIV-1 and Ebola virus particle release but is counteracted by the HIV-1 Vpu protein. Cell Host Microbe 2, 193-203 (2007)

9. Harila, K. et al. Vpu and Tsg101 regulate intracellular targeting of the human immunodeficiency virus type 1 core protein precursor Pr55gag. J. Virol. 80, 3765-3772 (2006)

10. Harila, K. et al. The Vpu-regulated endocytosis of HIV-1 Gag is clathrinindependent. Virology 369, 299-308 (2007)

11. Ishikawa, J. et al. Molecular cloning and chromosomal mapping of a bone marrow stromal cell surface gene, BST2, that may be involved in pre-B-cell growth. Genomics 26, 527-534 (1995).
12. Willey, R. L. et al. Human immunodeficiency virus type $1 \mathrm{~V}$ pu protein induces rapid degradation of CD4. J. Virol. 66, 7193-7200 (1992).

13. Ross, T. M., Oran, A. E. \& Cullen, B. R. Inhibition of HIV-1 progeny virion release by cell-surface CD4 is relieved by expression of the viral Nef protein. Curr. Biol. 9, 613-621 (1999).

14. Lama, J., Mangasarian, A. \& Trono, D. Cell-surface expression of CD4 reduces HIV-1 infectivity by blocking Env incorporation in a Nef- and Vpu-inhibitable manner. Curr. Biol. 9, 622-631 (1999).

15. Kupzig, S. et al. Bst-2/HM1.24 is a raft-associated apical membrane protein with an unusual topology. Traffic 4, 694-709 (2003).

16. Rollason, R. et al. Clathrin-mediated endocytosis of a lipid-raft-associated protein is mediated through a dual tyrosine motif. J. Cell Sci. 120, 3850-3858 (2007).

17. Jouvenet, N. et al. Plasma membrane is the site of productive HIV-1 particle assembly. PLoS Biol. 4, e435 (2006).

18. Muller, B. et al. Construction and characterization of a fluorescently labeled infectious human immunodeficiency virus type 1 derivative. J. Virol. 78, 10803-10813 (2004).

19. Goto, T. et al. A novel membrane antigen selectively expressed on terminally differentiated human B cells. Blood 84, 1922-1930 (1994).

20. Blasius, A. L. et al. Bone marrow stromal cell antigen 2 is a specific marker of type I IFN-producing cells in the naive mouse, but a promiscuous cell surface antigen following IFN stimulation. J. Immunol. 177, 3260-3265 (2006)

21. Brugger, B. et al. The HIV lipidome: a raft with an unusual composition. Proc. Natl Acad. Sci. USA 103, 2641-2646 (2006).

22. Bartee, E., McCormack, A. \& Fruh, K. Quantitative membrane proteomics reveals new cellular targets of viral immune modulators. PLOS Pathog. 2, e107 (2006).

Supplementary Information is linked to the online version of the paper at www.nature.com/nature.

Acknowledgements We thank members of the Bieniasz laboratory for comments and suggestions, P. Nahirney for assistance with electron microscopy, and $W$. Zhang and C. Zhao for assistance with the microarray experiments. This work was supported by grants from the NIH. P.D.B. is an Elizabeth Glaser Scientist of the Elizabeth Glaser Pediatric AIDS foundation.

Author Contributions S.J.D.N. and P.D.B conceived and designed the experiments. S.J.D.N. performed the experiments with assistance from T.Z. P.D.B. wrote the paper.

Author Information Reprints and permissions information is available at www.nature.com/reprints. Correspondence and requests for materials should be addressed to P.D.B (pbienias@adarc.org). 


\section{METHODS}

Cells and viruses. HeLa, HOS, 293T, HT1080, HeLa-TZMbl and Jurkat T cells were maintained using standard procedures. Human $\mathrm{CD} 4^{+} \mathrm{T}$ cells were isolated from whole blood using a commercially available kit (RosetteSep, StemCell Technologies) according to the manufacturer's instructions, and were $>90 \%$ pure before use (data not shown). The $\mathrm{CD}^{+} \mathrm{T}$ cells were stimulated with phytohaemagglutinin for 2 days, grown in the presence of IL-2 $\left(20 \mathrm{U} \mathrm{ml}^{-1}\right.$, Roche) for 3 days, and then grown for a further 2 days in the presence or absence of IFN- $\alpha\left(1,000 \mathrm{U} \mathrm{ml}^{-1}\right.$, Sigma).

HIV-1(WT) and HIV-1(delVpu) versions of the HIV-1 molecular clone NL4-3 have been previously described ${ }^{6}$, as have derivatives carrying YFP in the stalk region of matrix (HIV-1/MA-YFP) ${ }^{17}$. VSV-G-pseudotyped virus stocks were generated by transient transfection of 293T cells with proviral plasmid and pCMV-VSV-G complexed with polyethylenimine. Virus-containing supernatants were harvested $48 \mathrm{~h}$ after transfection, filtered, and infectious titres determined as $\beta$-galactosidase-positive colony-forming units using HeLaTZMbl indicator cells.

Microarray analyses. Total RNA was extracted, using TRIZOL (Invitrogen), from the various cell lines that were either untreated, or treated with $1,000 \mathrm{U} \mathrm{ml}^{-1} \mathrm{IFN}-\alpha$ for $24 \mathrm{~h}$ before harvest. cRNA was prepared and used to interrogate HumanRef- 8 v2 beadchip arrays containing 22,000 transcript probes (Illumina), according to the manufacturer's instructions. Relative expression levels in HeLa versus HOS cells, and IFN- $\alpha$-treated versus untreated 293T, HT1080 and Jurkat cells were calculated to identify tetherin candidates.

qRT-PCR. Total RNA (200 ng per assay) from untreated cells or cells treated with $1,000 \mathrm{U} \mathrm{ml}^{-1} \mathrm{IFN}-\alpha$ for $24 \mathrm{~h}$ before harvest was used to generate cDNA along with Superscript III reverse transcriptase (Invitrogen) and random hexamer primers. qRT-PCR was performed using a SYBR-green-containing master-mix and primer pairs specific for CD317 and GAPDH (Superarray Bioscience Corporation). Control reactions omitted reverse transcriptase, and the number of cDNA copies was determined using a cloned CDNA (tetherin) or a PCR-generated amplicon (GAPDH) as standards. Results (Fig. 1) were expressed as number of copies of $C D 317$ cDNA per copy of GAPDH cDNA.

Tetherin expression constructs and tetherin-expressing cell lines. PCR primers directed to the $5^{\prime}$ and $3^{\prime}$ ends of the $C D 317$ coding sequence were used along with HeLa-derived RNA to generate a CD317cDNA. This was inserted into pCR3.1/HA for transient expression and into the retroviral expression vector LHCX (Clontech) for stable expression of untagged, or N-terminally HAepitope-tagged forms of tetherin. The delCT and delGPI variants of tetherin which lacked the $\mathrm{N}$-terminal 20 amino acids and C-terminal 19 amino acids, respectively, were constructed using PCR primers directed to the corresponding sequences. 293T- and HT1080-derived cell lines stably expressing untagged or HA-tagged tetherin were derived by transduction with LHCX/tetherin retrovirus stocks. Transduced cells were selected in hygromycin $\left(100 \mu \mathrm{g} \mathrm{ml}^{-1}\right)$ and were used as a pool.

Transfection-based virion release assays. $293 \mathrm{~T}$ cells in 24 -well plates $\left(1 \times 10^{5}\right)$ were transfected with HIV-1(WT) or HIV-1(delVpu) proviral plasmids, along with varying quantities of a PCR3.1/HA-based tetherin expression plasmid using Lipofectamine 2000 (Invitrogen). Alternatively, unmodified or stable tetherinexpressing 293T cells were transfected with plasmids expressing MLV GagPol in the presence or absence of co-expressed Vpu. At $48 \mathrm{~h}$ after transfection, cells and culture supernatants were collected for analysis (see below).
Single-cycle HIV-1 replication assays. HeLa, 293T, or HT1080 cells in 24-well $\left(5 \times 10^{4}\right)$ or 12 -well $\left(1 \times 10^{5}\right)$ plates were infected with VSV-G-pseudotyped HIV-1(WT) or HIV-1(delVpu) stocks at 0.2 infectious units per cell and the inoculum removed $6 \mathrm{~h}$ later. At $48 \mathrm{~h}$ after infection, culture supernatants were harvested, and infected cells lysed for analysis of released virion yield and viral protein expression. In experiments involving tetherin depletion, HeLa cells were transfected twice (at $24 \mathrm{~h}$ and $6 \mathrm{~h}$ before infection) with a pool of siRNAs targeting tetherin (Dharmacon Smartpool, catalogue number L-011817) or a control siRNA pool (Dharmacon Smartpool control, catalogue number D-001210-01-20).

Analysis of protein expression and virus release. Culture supernatants from infected or transfected cells were clarified by low-speed centrifugation, passed through a $0.22 \mu \mathrm{m}$ filter and virion particles were pelleted through a $20 \%$ sucrose layer at $30,000 \mathrm{~g}$. Virus particle pellets and corresponding cell lysates were analysed by SDS-PAGE and western blot assays using anti-HIV capsid (p24), anti-MLV capsid (p30) and/or anti-HA monoclonal antibodies and anti-mouseperoxidase antibodies, as previously described ${ }^{6,8}$.

Infectious virus yields were determined using clarified culture supernatants. HeLa-TZMbl cells, which express CD4, CXCR4 and CCR5 and contain an integrated lac $Z$ reporter gene under the control of an HIV-1 LTR, were inoculated with serially diluted supernatants for $4 \mathrm{~h}$ and washed. Thereafter, $\beta$-galactosidase activity in lysates of the infected indicator cells was determined $48 \mathrm{~h}$ later using GalactoStar reagent (Tropix), according to the manufacturer's instructions.

Protease stripping assays. 293T cells stably expressing tetherin were transfected with HIV-1(WT) or HIV-1(delVpu) proviral plasmids, either alone or along with a plasmid expressing cherry-FP-Rab5a $(\mathrm{S} 34 \mathrm{~N})$. Constitutively released particles were harvested from culture supernatants. Thereafter, cells were incubated for $15 \mathrm{~min}$ at $37^{\circ} \mathrm{C}$ in buffer $(100 \mu$ of Tris/ $\mathrm{HCl}(\mathrm{pH} 8.0), 150 \mathrm{mM}$ $\mathrm{NaCl}, 5 \mathrm{mM} \mathrm{CaCl}_{2}$ ) with or without the addition of subtilisin $\left(1 \mathrm{mg} \mathrm{ml}^{-1}\right)$. The stripping reaction was stopped with $0.5 \mathrm{ml}$ DMEM/FCS containing $5 \mathrm{mM}$ PMSF. Cells were pelleted and the supernatants were filtered $(0.2 \mu \mathrm{m})$ before harvesting the released virion particles, by pelleting through sucrose, as described above.

Microscopy. 293T or HT1080 cells that were either unmodified or stably expressed untagged or HA-tagged tetherin were plated on coverslips and transiently transfected with various combinations of plasmids expressing HIV-1 Gag-GFP, untagged Vpu, Vpu-YFP or Cherry-FP-Rab5a mutant (S34N or Q79L). Alternatively, cells were infected, as described above, with VSV-Gpseudotyped HIV-1/MA-YFP derivatives at 0.2 infectious units per cell. At $18 \mathrm{~h}$ after transfection, or $48 \mathrm{~h}$ after infection, cells were fixed with $4 \%$ paraformaldehyde. Anti-HA or anti-CD63 antibodies were used to stain HA-tetherin and late endosomal compartments, respectively, followed by an anti-mouse Alexa Fluor 595 conjugate. Microscopy was done using a Deltavision suite (Applied Precision) as described previously ${ }^{6,17}$.

For electron microscopy, HT1080 cells that were unmodified or stably expressed tetherin were infected with VSV-G-pseudotyped HIV-1(WT) or HIV-1(delVpu) at approximately five infectious units per cell. At $48 \mathrm{~h}$ after infection, cells were fixed in $2 \%$ glutaraldehyde $/ 2 \%$ paraformaldehyde in sodium cacodylate buffer, embedded in gelatin, post fixed in $1 \%$ osmium tetroxide, ethanol dehydrated and embedded in Spurr's resin. Sections $(100 \mathrm{~nm})$ were stained with uranyl acetate and lead citrate and observed with a FEI G2 Tecnai TEM at $80 \mathrm{kV}$ equipped with a $4 \mathrm{~K} \times 4 \mathrm{~K}$ Gatan digital camera. 